\title{
AUTONOMOUS OCEANOGRAPHIC SAMPLING NETWORKS
}

\author{
Thomas B. Curtin, James G. Bellingham, Josko Catipovic and Doug Webb
}

\section{Spatially adaptive \\ sampling is necessary}

to resolve evolving

gradients with

sparsely distributed

sensors.

\begin{abstract}
A ssessing the reality of numerical model fields with ever increasing resolution, testing dynamical balances involving high-order derivatives, and exploring the limits of predictability require measurement of temporal and spatial gradients in the ocean far exceeding current practical capabilities. Sensors for various properties have improved steadily in recent years, capitalizing on advances in electronics. fiber optics, and materials. In contrast. platforms enabling measurement of property gradients remain primitive. With current trends, advances in a large class of ocean science problems will be increasingly platform limited.

One example in this class is frontal dynamics where cross-front circulation must be resolved to assess nonlinearity. dissipation and biomass, and along-front shear must be determined to examine instability mechanisms. Other examples include surface-layer dynamics where solar, wind, and wave energies produce Langmuir circulation, mixing, subduction and patchy primary productivity: stratified turbulence that may be organized by internal waves and mesoscale shear; cross-shelf transport, a complex resultant of tidal. wind and buoyancy forces; deep convection, an intermittent and three-dimensional process resulting from preconditioning and local fluxes; and sea ice mass balance involving both the extent and thickness fields. There are also applied problems such as providing surface truth for satellite remote sensing, measuring open boundary conditions for regional forecast models, mapping of properties in remote areas, and monitoring pollutants.

Current sampling is done primarily from ships, satellites. floats, and moorings. The most comprehensive observations from ships involve underway profilers such as thermistor chains, acoustic
\end{abstract}

T.B. Curtin. Office of Naval Research, 800 North Quincy St.. Arlington, VA 22217-5000, USA: J.G. Bellingham, Massachusetts Institute of Technology. Cambridge. MA 02139. USA: J. Catipovic, Woods Hole Oceanographic Institution. Woods Hole. MA (2543, USA: D. Webb, Webb Research Corporation, Falmouth. MA 02536. doppler current profilers, and undulating towed bodies. These methods provide quasi-synoptic, two-dimensional sections through evolving fields. Satellites also provide two-dimensional realizations of the ocean surface, although inferences about three-dimensional structure can be made. Increasing spatial resolution from ships and satellites generally introduces temporal aliasing when the number of platforms is limited. Arrays of moorings and floats provide simultaneous time series, but spatial sampling is typically sparse due to cost. Spatially adaptive sampling is necessary to resolve evolving gradients with sparsely distributed sensors. Long duration, high resolution and affordability dictate a robust, distributed, autonomous system with low unit cost.

\section{Overall System Concept}

An approach toward four-dimensional ocean sampling is the Autonomous Oceanographic Sampling Network (AOSN), depicted in a coastal frontal zone (Fig. 1). Sampling of the high gradients associated with the front is done with several autonomous underwater vehicles (AUVs) as well as with distributed acoustic and point sensors. The objective is to combine the best features of each method for increased mapping resolution. The vehicles traverse the network recording temperature, salinity. velocity. and other data. relaying key observations to the network nodes in real time and transferring more complete data sets after docking at a node. Each network node (Fig. 2) consists of a base buoy or mooring containing an acoustic beacon, an acoustic modem, point sensors, an energy source and a selectable number of AUV docks. Acoustic transmission loss along the many internodal paths is measured periodically. A central location, either one of the nodes and/or onshore, processes the information in near real time to guide vehicle sampling. For example, the coastal front is localized acoustically every hour. The network controller dispatches vehicles to frontal regions indicating gradient intensification for detailed cross-front and along-front measure- 


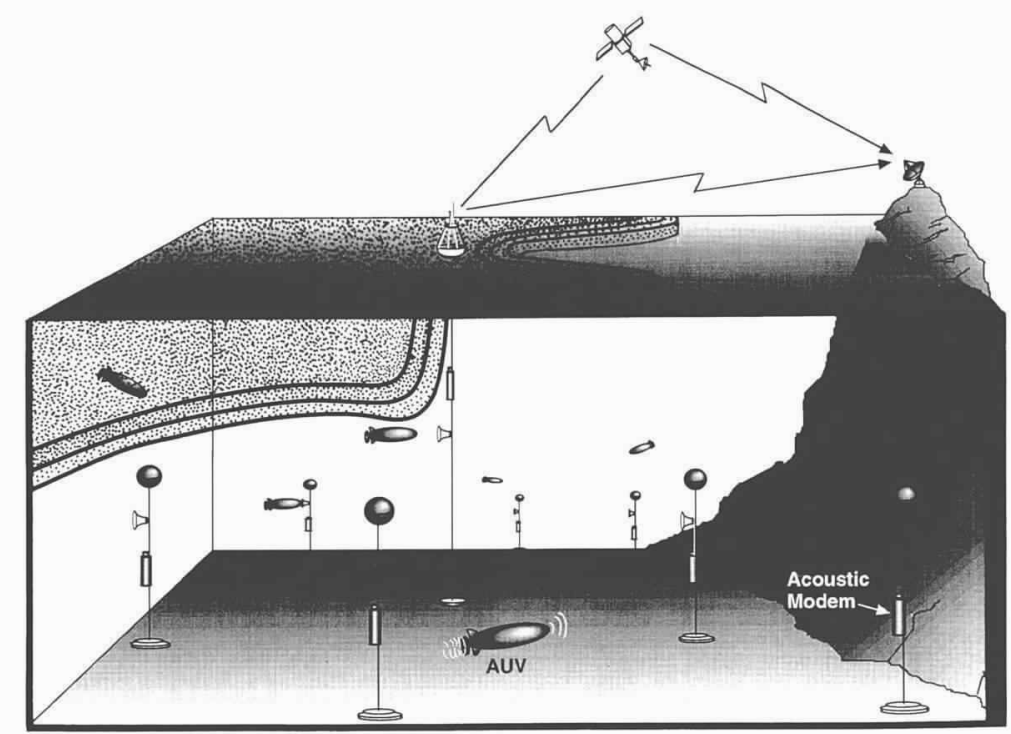

Autonomous Oceanogra!phic Semsoling Network (AOSN)

Fig. 1: An Autonomous Oceanographic Sampling Network (AOSN) deployed in a coastal ocean frontal regime. Each network node consists of a base buoy and a selectable number of small Autonomous Underwater Vehicles (AUVs).

ments to determine the nature and extent of developing instabilities. The vehicles alter their paths in response to both locally sensed gradients and the evolving global data set. The strategy is to bound errors in measured gradient fields to limits acceptable for specific hypothesis tests. Key network advantages include synoptic volume coverage, adaptive sampling, flexible control, energy management, and robustness to component failure.
The practicality of this concept rests on the number of AUVs required, the type of AUV, and the performance of acoustic navigation and telemetry.

\section{Vehicle Population}

To obtain synoptic data, a survey system must be capable of mapping an ocean structure faster than significant changes occur in that structure. For the coastal front example, tidal forcing may
.. vehicles alter

their paths in response to both locally sensed gradients and the evolving global data set.

\section{NETWORK NODES}

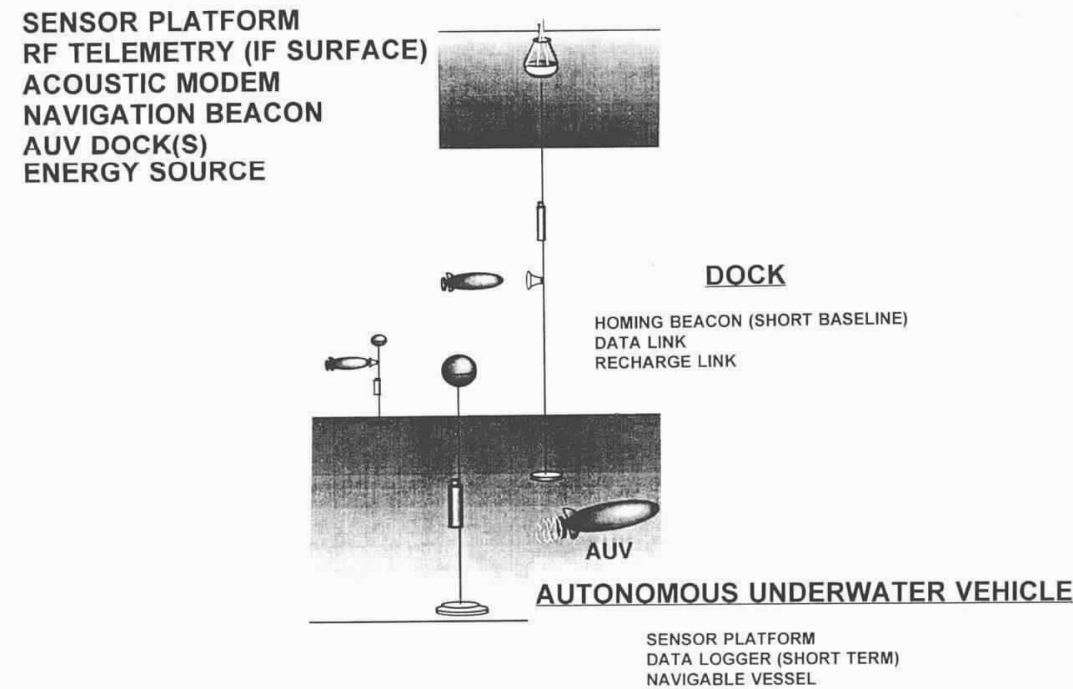

Fig. 2: Components and functions of a network node. For RF telemetry to shore, only one node in the network need have a surface expression. 
... the larger the

vehicle number, the

lower the expenditure

of energy by a single

vehicle. dictate a complete survey every 6 hours and typical cross-front gradients require a minimum spatial resolution of $0.5 \mathrm{~km}$. If a survey over an area A must be completed in time $t$ with a resolution $L$. then the required effective survey velocity $\left(V_{c}\right)$ is

$$
\left.\mathrm{V}_{\mathrm{e}}=1 / \mathrm{t}[(\mathrm{A} / 2 \mathrm{~L})-2 \mathrm{~L})\right] \approx \mathrm{A} /(2 \mathrm{~L} \mathrm{t})
$$

The resolution, $\mathrm{L}$, is defined here as the maximum horizontal distance any point in the area is from a survey track. For the front, a representative survey volume is $500 \mathrm{~km}^{2}$ in surface area and $200 \mathrm{~m}$ deep. Given the 6-hour survey time and the 0.5 $\mathrm{km}$ desired resolution, the effective survey velocity is $83 \mathrm{~km} / \mathrm{hr}(23 \mathrm{~m} / \mathrm{s})$.

Energy storage has long been a problem for remote oceanographic systems. Consequently, an important figure of merit for an autonomous survey system is the energy required per kilometer covered. An advantage of a multiple vehicle survey system is that the effective survey velocity for each vehicle is reduced in proportion to the number of vehicles, leading to increased efficiency. The power consumed by an AUV is

$$
\mathrm{P}=\left(\mathrm{rDSV} V^{3}\right) /(2 \mathrm{~h})+\mathrm{H}
$$

where $\mathrm{P}$ is total power consumed, $\mathrm{h}$ is efficiency of propulsion, $\mathrm{r}$ is density, $\mathrm{D}$ is drag coefficient, $\mathrm{S}$ is vehicle surface area. $V$ is vehicle velocity, and $\mathrm{H}$ is hotel load (power used by the vehicle for functions other than propulsion). The total energy required per unit distance of track covered, E, is

$$
\mathrm{E}=[(\mathrm{rDS}) /(2 \mathrm{~h})]\left(\mathrm{V}_{\mathrm{e}} / \mathrm{N}\right)^{2}+(\mathrm{NH}) / \mathrm{V}_{\mathrm{e}}
$$

A

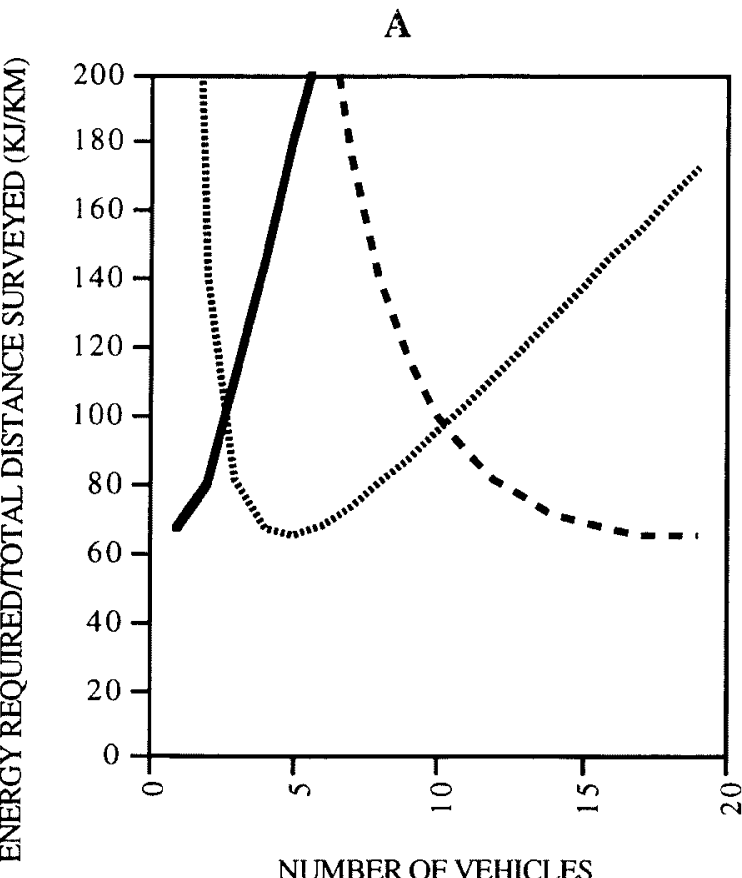

NUMBER OF VEHICLES where $\mathrm{N}$ is the number of vehicles. This relationship demonstrates two trade-offs in minimizing required energy: decreasing the energy consumed by the combined vehicle hotel loads drives the number of vehicles down, while decreasing energy consumed by propulsion drives the number of vehicles up. Joules required per kilometer of coverage are shown in Figure 3 as a function of the number of vehicles. With the inverse quadratic dependence of drag-related energy consumption on vehicle number, the penalty for too few vehicles is greater than the penalty for too many vehicles, especially at low hotel loads. Clear benefit is obtained by decreasing the hotel load, justifying the effort to minimize power use by vehicle subsystems.

Using the relationships defined above, an optimum number of vehicles, $\mathrm{N}_{\text {op }}$. can be determined for surveying a given phenomenon. Setting the derivative of the energy equation above to zero.

$$
N_{\text {op }}=[(\mathrm{rDS}) /(\mathrm{hH})]^{1 / 3}[\mathrm{~A} /(2 \mathrm{Lt})]
$$

The optimum number of vehicles varies with the area of the region surveyed and inversely with the required resolution and completion time. The optimum vehicle number increases weakly as vehicle size increases and as hotel load decreases. An assumption in the analysis is that the vehicle size remains constant. For the same total track coverage. the larger the vehicle number, the lower the expenditure of energy by a single vehicle. Therefore. smaller vehicles are possible as more vehicles are used, tilting the energy efficiency arguments even further in favor of multiple vehicles.

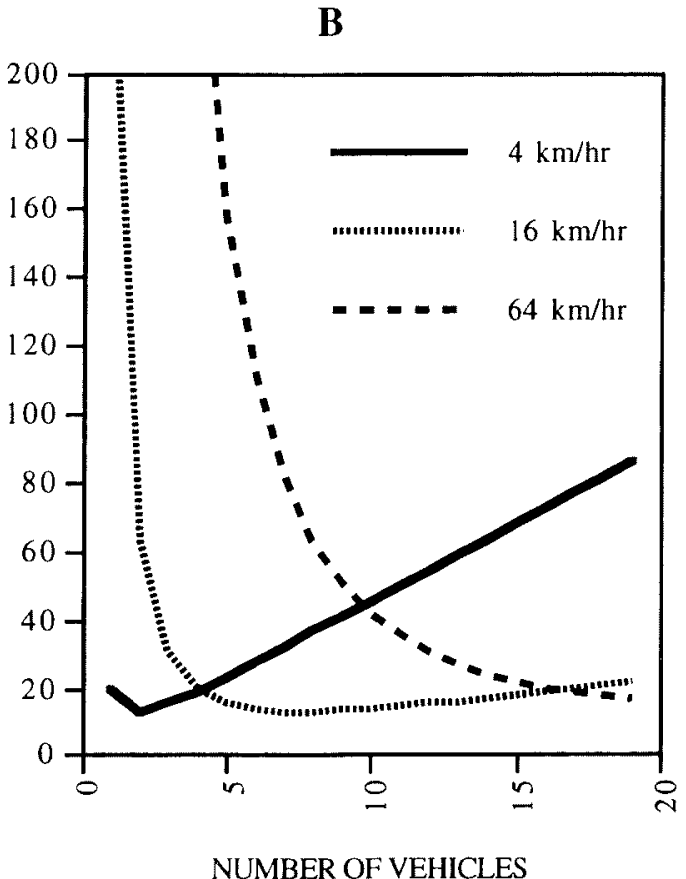

Fig. 3: Energy per kilometer of transit for typical small class vehicles with propulsion efficiency of 35\% and hotel load of $10 \mathrm{~W}(\boldsymbol{A})$ and propulsion efficiency of $70 \%$ and hotel load of $5 \mathrm{~W}(\boldsymbol{B})$. 


\section{Vehicle Class}

The essence of the approach here is deployment of a flexible network of many low-cost lightweight vehicles with reliable navigational skills versus using a few expensive very sophisticated vehicles. To achieve the design goals and produce a system of practical use to the oceanographic community, AUV cost must remain within a critical cost-size envelope. Large vehicles $(>10 \mathrm{~m}$ long, $1 \mathrm{~m}$ diameter) are too costly to build and operate; small vehicles $(<1 \mathrm{~m}$ long, $0.1 \mathrm{~m}$ diameter) are too inflexible in accepting off-the-shelf sensors and computer hardware. The best vehicle for the AOSN is moderate in size $(1-3 \mathrm{~m}$ long, $0.2-0.8 \mathrm{~m}$ diameter) and cost ( $\$ 10 \mathrm{~K}$ to $\$ 50 \mathrm{~K})$, yet capable of carrying oceanographic sensors over ranges of at least hundreds of kilometers. Vehicles in this class also have a variety of value-added advantages compared with larger vehicles. They can be deployed and recovered in rougher seas and off smaller vessels. Low cost manufacturing techniques can be employed. They are more maneuverable in confined environments or near bottom. Higher thrust-to-mass ratios can be obtained for better control in current shear and turbulence. They are robust to collisions and cause less damage. To keep costs low and reliability high, a modular design is necessary. Modularity is equally important for electronics (hardware), "intelligence" (software), and mechanical construction.

The AOSN class of high performance interactive AUVs is driven by ocean synoptic sampling requirements, a generically different design problem than has been addressed by most AUV researchers. Existing military vehicles (e.g.. ARPA vehicles, MUST, and AUSS) are for the most part large (weighing several tons) and high cost $(>\$ 1 M)$. Vehicles designed for scientific research (e.g., EAVE, NPS-2, and VORTEX) are typically single-unit testbeds operated in limited environments. Large, relatively high cost vehicles (e.g., DOLPHIN and DOGGIE) intended to replace single research vessels are also being developed. The many remotely operated vehicles (ROVs) available are not designed for autonomous, coordinated operation. AUVs in the class necessary for AOSNs are just now being developed.

An example of a prototype small high-performance propeller-driven vehicle in this class is Odyssey (Fig. 4; Bellingham et al., 1992). Odyssey is a mobile instrumentation platform with actuators, sensors, and on-board intelligence designed to complete sampling tasks autonomously. Odyssey is $2.1 \mathrm{~m}$ long, has a maximum diameter of $0.6 \mathrm{~m}$, and is rated to a depth of $6,700 \mathrm{~m}$. A freeflooded plastic shell shaped by vacuum forming provides a low-drag hull with a cost under $\$ 1 \mathrm{~K}$. Inside the hull is a selectable pressure case (standard glass instrumentation spheres for deep ocean work) and a variety of subsystems such as water property sensors, propulsion motor, control surface actuators, and sonar transducers. Figure 5 shows range versus speed curves $(h=0.35$ and $D$ $=0.005$ at $1.5 \mathrm{~m} / \mathrm{s}$ ). Note that as hotel load decreases, the maximum range is achieved at lower speed. Odyssey has been deployed in a variety of settings, most recently off Antarctica from the icebreaker Nathaniel B. Palmer.

Buoyancy-driven AUVs are also practical and indicated for some objectives. An example is the Slocum vehicle (Fig. 6), a small, almost neutrally buoyant glider that moves vertically and horizontally through the water as a result of small changes in buoyancy. Two versions of Slocum are under development. For the open temperate and tropical ocean, Slocum derives its propulsion

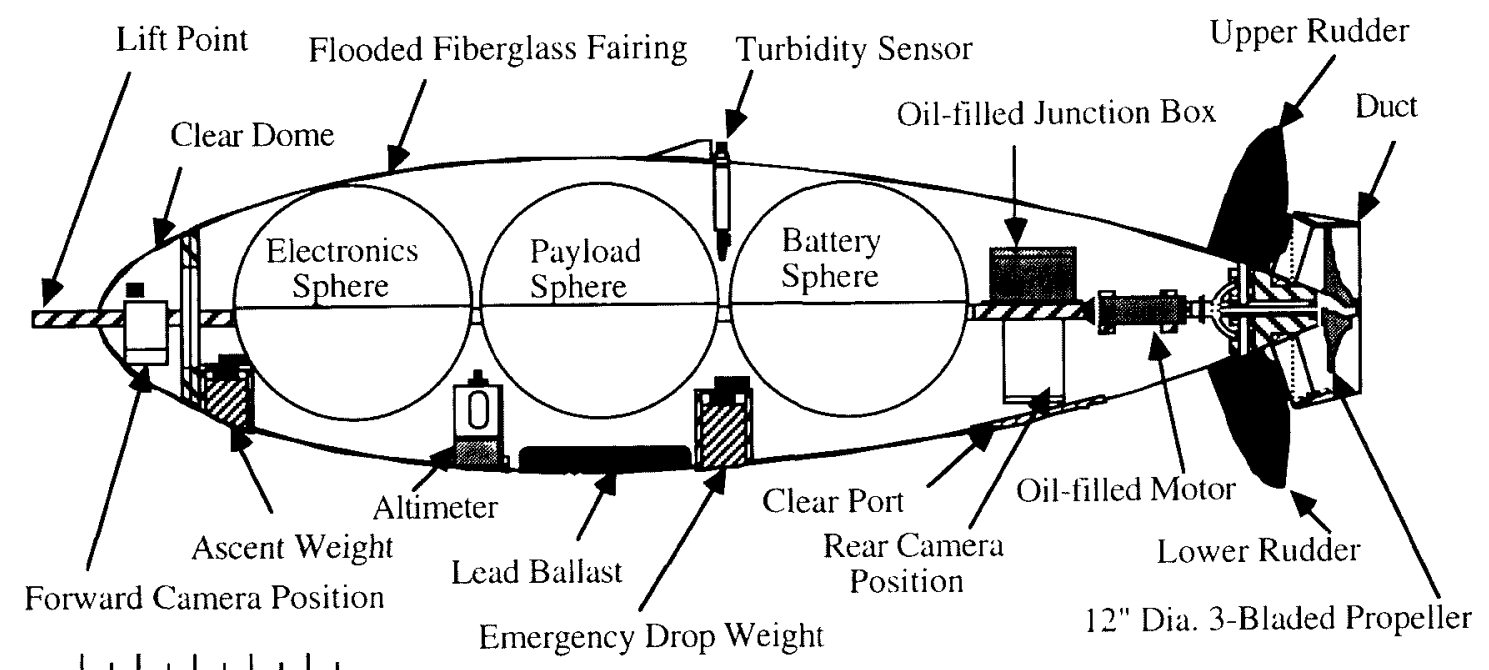

$\frac{L}{0} \frac{1}{10}+\frac{1}{20}+\frac{1}{30}+\frac{1}{40} \mathrm{~cm}$.

Fig. 4: Schematic of Odyssey. The outer faired surface is a low drag form. A ducted propeller is used to minimize fouling. Steering is by cruciform control surfaces. 


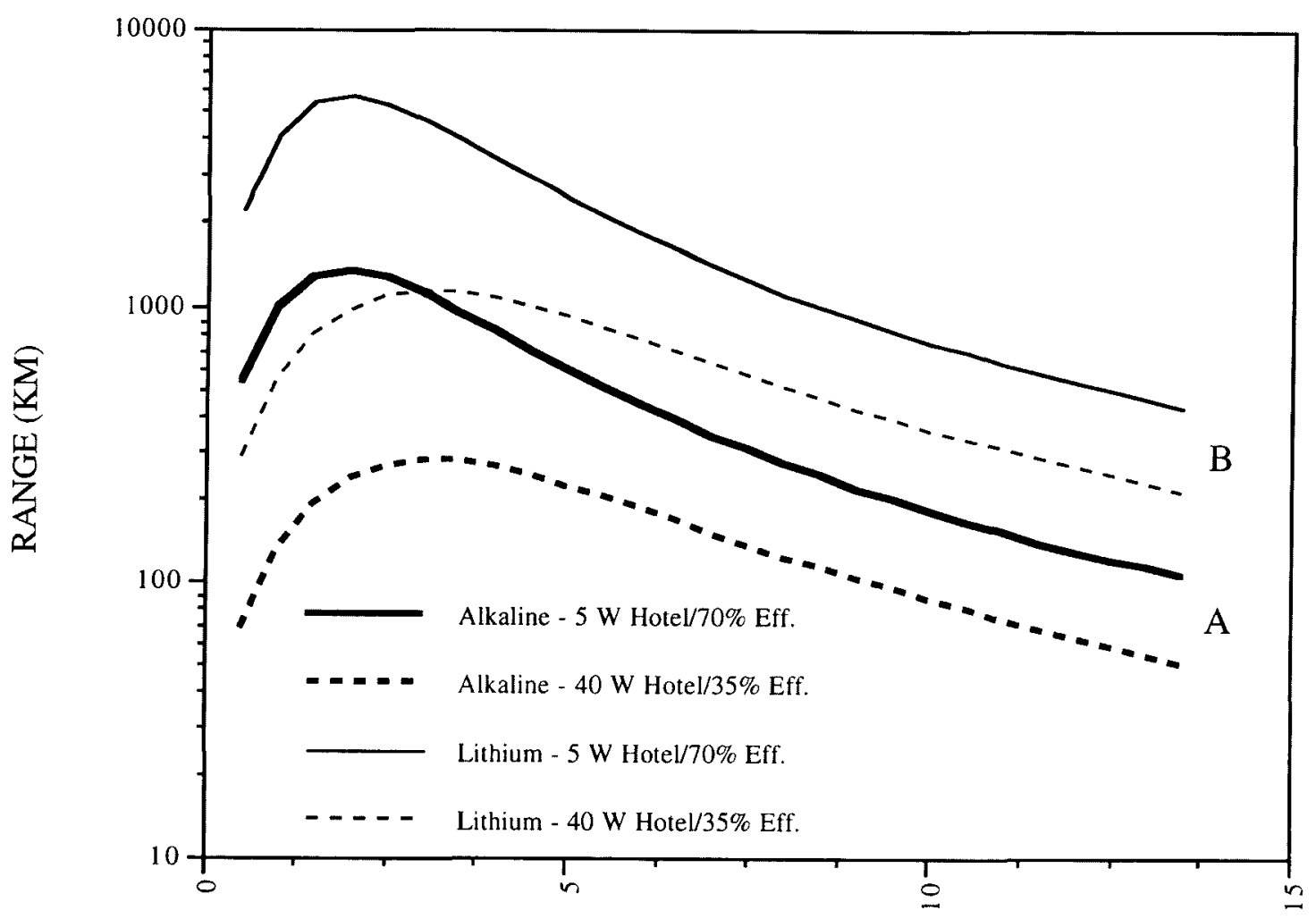

\section{SPEED (KM/HR)}

Fig. 5: Odyssey range as a function of speed for two different hotel loads and $50 \mathrm{~kg}$ of alkaline-manganese dioxide batteries (Duracell D Cells) $(A)$ and lithium-thionyl chloride batteries [BEltype 64$152(\# 6)](B)$. Battery temperature is $0^{\circ} \mathrm{C}$. Typical hotel loads are $51 \mathrm{~W}$ average and $92 \mathrm{~W}$ peak.

\section{$A_{\text {key issue is }}$ \\ energy efficiency, \\ which varies from \\ $1 \mathrm{kbit} / \mathrm{joule}$ per $\mathrm{km}$ \\ . . . to $100 \mathrm{kbit}$}

joule per $\mathrm{km}$. . . power for a $2,000 \mathrm{~m}$ deep sawtooth ascent/descent from a unique heat engine that utilizes heat flow from the warm surface to the cool deep water. By exploiting thermal energy from the environment, a 5-year, $40,000-\mathrm{km}$ duration is feasible. An interactive network of such vehicles is particularly suitable for sampling large-scale ocean processes. In areas of inadequate temperature differences to provide power for propulsion, such as shelf waters, the same vehicle is fitted with a battery-operated buoyancy controller (similar to that used in ALACE) and can operate with a 40 -day, $800-\mathrm{km}$ duration. Slocum's are slow speed AUVs and must be used with care in high-current areas. They are quiet but not silent in operation. High efficiency (no external control surfaces) but slow rate maneuverability has been achieved with internal control of the center of gravity. This class of vehicle is poor at precision docking but excellent at station keeping within a given area. for example. sampling for years within a few kilometers of a fixed position.

\section{Acoustic Telemetry and Navigation}

The network is linked by what is in effect an underwater acoustic cellular telephone system with modems on AUVs and at buoy nodes. The nodes communicate among themselves and establish protocols for information routing in response to changes in acoustic paths and ambient noise much as cellular telephone routing stations establish means for locating and communicating with users. State-of-the-art acoustic modems are capable of $10 \mathrm{kbit} / \mathrm{s}$ data rates at $10 \mathrm{~km}$ range and 3 $\mathrm{kbit} / \mathrm{s}$ at $90 \mathrm{~km}$ (Catipovic et al., 1994). These ranges and data rates enable efficient telemetry of commands to AUVs and also transfer of large amounts of recorded data from the vehicles to the nodes. A key issue is energy efficiency, which varies from $1 \mathrm{kbit} / \mathrm{joule}$ per $\mathrm{km}$ with off-the-shelf commercial modems (e.g., Datasonics Inc.) to 100 $\mathrm{kbit} / \mathrm{joule}$ per $\mathrm{km}$ for research prototypes. Such efficiency is manageable on small AUVs.

A complete AOSN system includes a radio frequency (RF) link to the user, either via satellite for long ranges or a line-of-sight RF modem for ranges of $10-20 \mathrm{~km}$. The currently available ARGOS system will soon be supplemented by low-earth-orbiting microsats, commercial communication satellites (e.g., at C-band), and/or the global cellular telephone satellites (Iridium), all with much higher data rates and downlink capabilities. For coastal work, $920 \mathrm{MHz}$ digital RF systems are capable of transmitting from $200 \mathrm{kbit} / \mathrm{s}$ to 


\section{SLOCUM Glider}

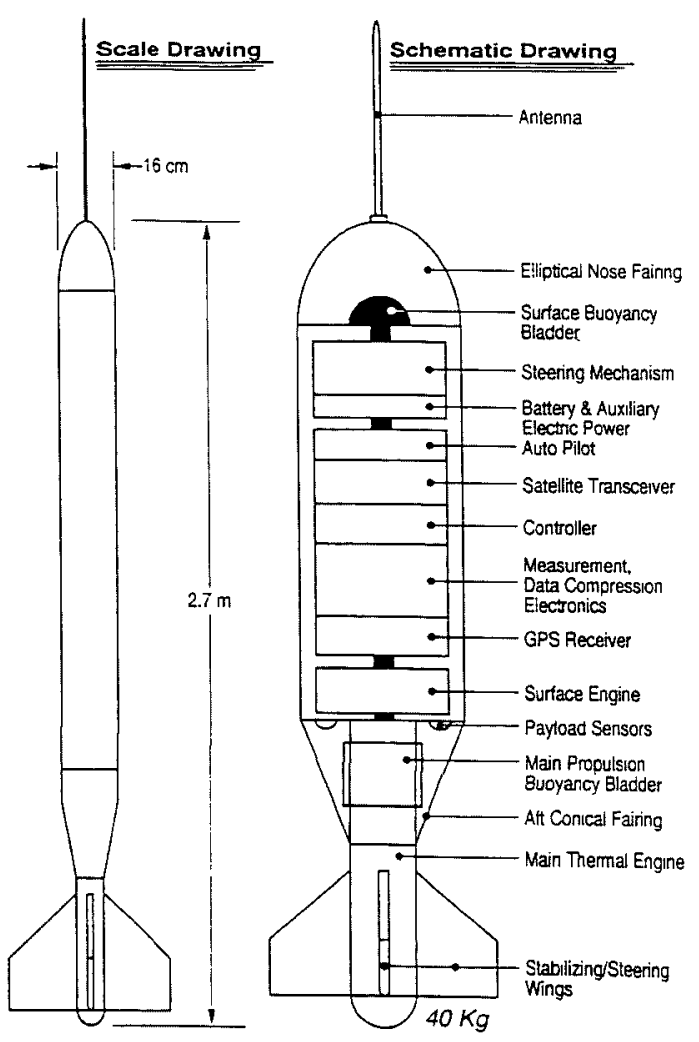

Fig. 6: Schematic of Slocum, a small almost neutrally buoyant glider that moves vertically and horizontally through the water driven by small changes in buoyancy. Steering is by control surfaces or internal center of gravity adjustment.

$2 \mathrm{Mbit} / \mathrm{s}$ at modest power levels using familiar TCP-IP protocols. This allows simple interfacing to commercially available workstations for data display, archiving, and adaptive experiment control.

An Acoustic Local Area Network (ALAN) is presently deployed in Monterey Canyon connecting ocean bottom seismometers and current meters to Internet, enabling real-time access to in situ instrumentation. An integrated acoustic telemetryAUV network is scheduled for spring deployment in the Arctic as part of an Ice Mechanics experiment (Fig. 7). Engineering goals are to demonstrate network operation in high latitude acoustic environments and to integrate the Odyssey into network operation. The Arctic system will consist of six compact through-ice buoys with RF links in air and an acoustic network in the water. One goal is to determine maximum internode spacing that yields robust communication. In addition, the turbulence structure in the under-ice boundary layer will be detected and mapped. Following the Arctic experiment, a deployment on the continental shelf is planned that will demonstrate autonomous acoustic message routing in the difficult shallow water environment (Fig. 8).

For navigation, no single technique provides a broadly applicable solution for small, long-range AUVs. A variety of technologies exist today. including radio and satellite systems, long, short and ultrashort baseline acoustic systems (Abbott, 1978; Elliot and Olson, 1984; Jacobsen et al., 1985), acoustic doppler and correlation speed logs (Sternick, 1978; Dickey and Edward, 1978), inertial systems (Johnstone and Fries, 1988) and terrain-following techniques (Stasior, 1991; Tuohy et al., 1993). Radio and satellite navigation requires that the vehicle be at or close to the surface, long baseline acoustic navigation accuracy degrades dramatically at longer ranges, and inertial systems have relatively high error levels for slow-moving AUVs. Combining complementary techniques is one way to achieve improved performance, for example, using both Doppler sonar and inertial navigation (Hutchison, 1991). For acoustic navigation systems, accuracy is being iteratively enhanced in the network environment through timely feedback of the measured sound speed field into onboard acoustic propagation models. In the Arctic, a location precision of $1 \mathrm{~m}$ over a range of $10 \mathrm{~km}$ can be achieved by capitalizing on multiple arrivals from navigation beacons (Deffenbaugh, 1993).

\section{Enabling Technologies}

Much of the underlying technology required for the AOSN now exists or is being rapidly developed. Engineering is needed to interface components and optimize performance for small smart AUVs. A number of technologies, discussed below, are "high leverage"; advances in these areas will substantially improve AOSN capabilities. As network-based sampling evolves, its most important component will shift from the hardware elements to the software operating system.

\section{Intelligent Control}

Software for intelligent control of AUVs remains a challenging research area. Any successful approach must be anchored in the real world of limited computational resources, imprecise sensor data, unpredictable subsystems, and the high cost of program development time. The objective is to develop vehicles that are rugged and capable of complex missions with abstract goals, for example, to find and map extrema in the convergence field within the coastal frontal zone (Fig. 1). Capabilities that will become part of a vehicle's intelligence include rendezvous and docking, survey, gradient following, obstacle avoidance, adaptive sampling, terrain following, and fault detection and recovery. For the latter, both hardware faults (e.g., failure of a thruster) and procedural failures (e.g. missed approaches on docking) must be managed. Reconfiguring vehicle software for a range of missions, sensor types and performance
Combining

complementary

techniques is one

way to achieve

improved

performance, . . . 


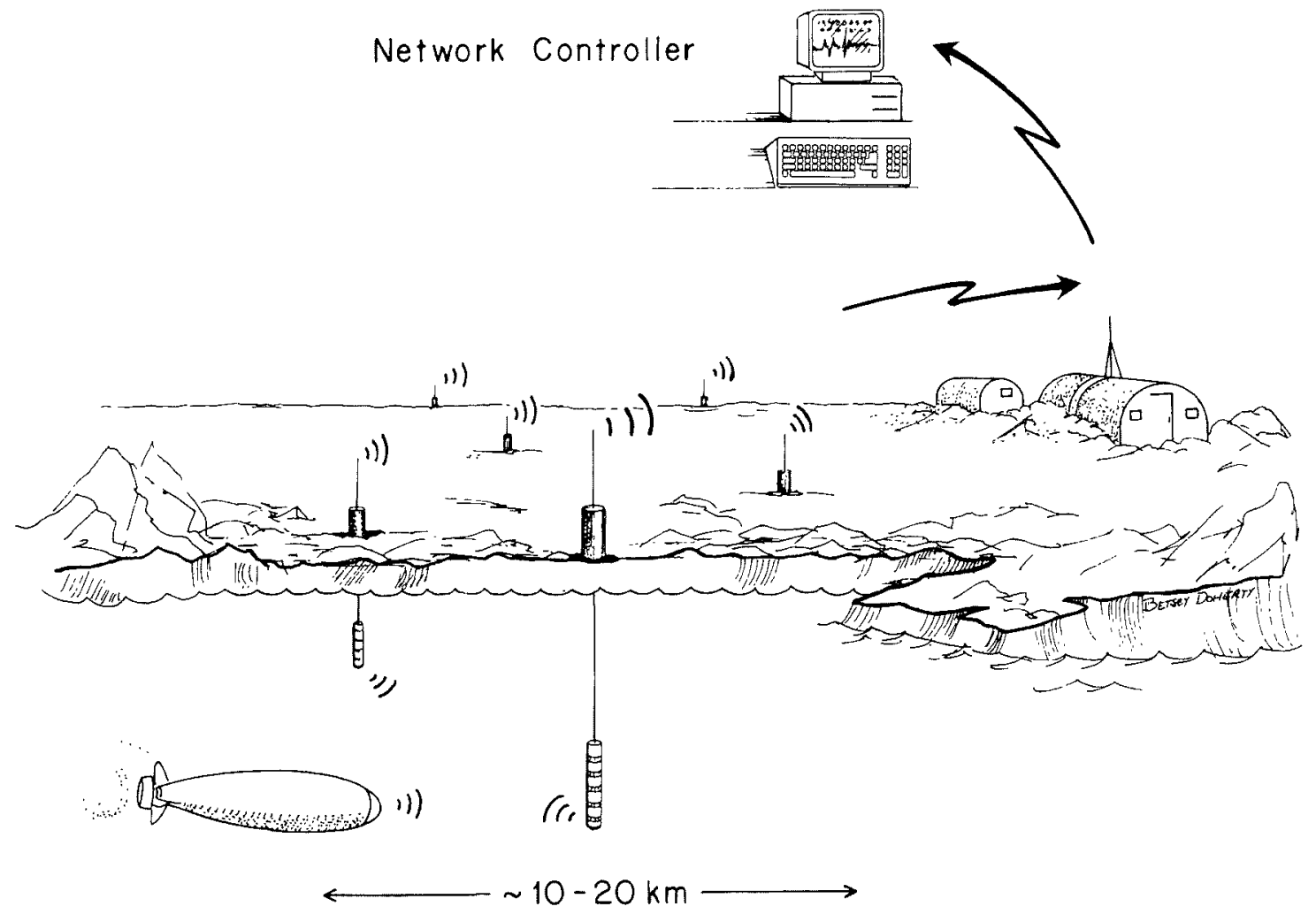

Fig. 7: Schematic of an underice Acoustic Local Area Network (ALAN) to be deployed together with an AUV in the Beaufort Sea in spring 1994. Objectives include mapping of underice morphology and ocean boundary layer properties.

At present, only

electric batteries are

practical for small,

inexpensive AUVs, ... must be straightforward, since the reconfiguration will often be accomplished remotely through one of the RF-linked nodes or through an acoustic link.

Efficiencies in coordinating multiple vehicles and managing the system for maximum endurance are also important. As the number of vehicles increases, the aggregate ratio of sampling time to docking and transit time changes. In sampling the vertical structure of the frontal zone, trade-offs between small numbers of vehicles executing high amplitude yo-yo tracks and greater numbers tracking smaller vertical excursions must be carefully analyzed. Intelligent control of multiple vehicle operations involves communication protocols. decision priorities, adaptive responses, distribution of control authority, relative navigation and sensor fusion (Albus, 1988; Turner et al., 1988; Triantafyllou et al., 1991).

\section{Data Management}

New sensor technology on AUVs supporting high-resolution science objectives will challenge onboard data management. Traditional methods probably will be inadequate. Two central problems in autonomous sensing are the representation of a particular data type and the management of uncertainty. The utility of continuous, nonlinear, higher-dimensional geometry in representing database information is being explored (Patrikalakis and Bardis, 1991). Such approaches are attractive in environments where gradients are of primary interest. Although the computational resources available on future AUVs will be formidable, effective encoding of geophysical fields including error fields and their efficient update and interrogation will require new approaches.

\section{Energy Storage and Power Management}

The network approach greatly reduces energy limitations through both platform diversity and cache storage capacity at docks. Although energy capacity $(\mathrm{W}-\mathrm{hr} / \mathrm{kg})$ of an individual vehicle is an important variable, large energy capacity is useless without the required power delivery $(\mathrm{W} / \mathrm{kg}$ ). At present, only electric batteries are practical for small, inexpensive AUVs, although other energy storage systems (e.g.. fuel cells) may prove competitive. Most scientific sampling objectives can be achieved within the limitations of state-of-theart battery technology by adjusting variables (e.g., number of nodes, number of vehicles, duty cycle, and trackline traverse relative to existing currents). Cells with known recharge behavior, noncatastrophic failure (e.g., thermal runaway) and capacity at low temperature are desirable. Further ad- 

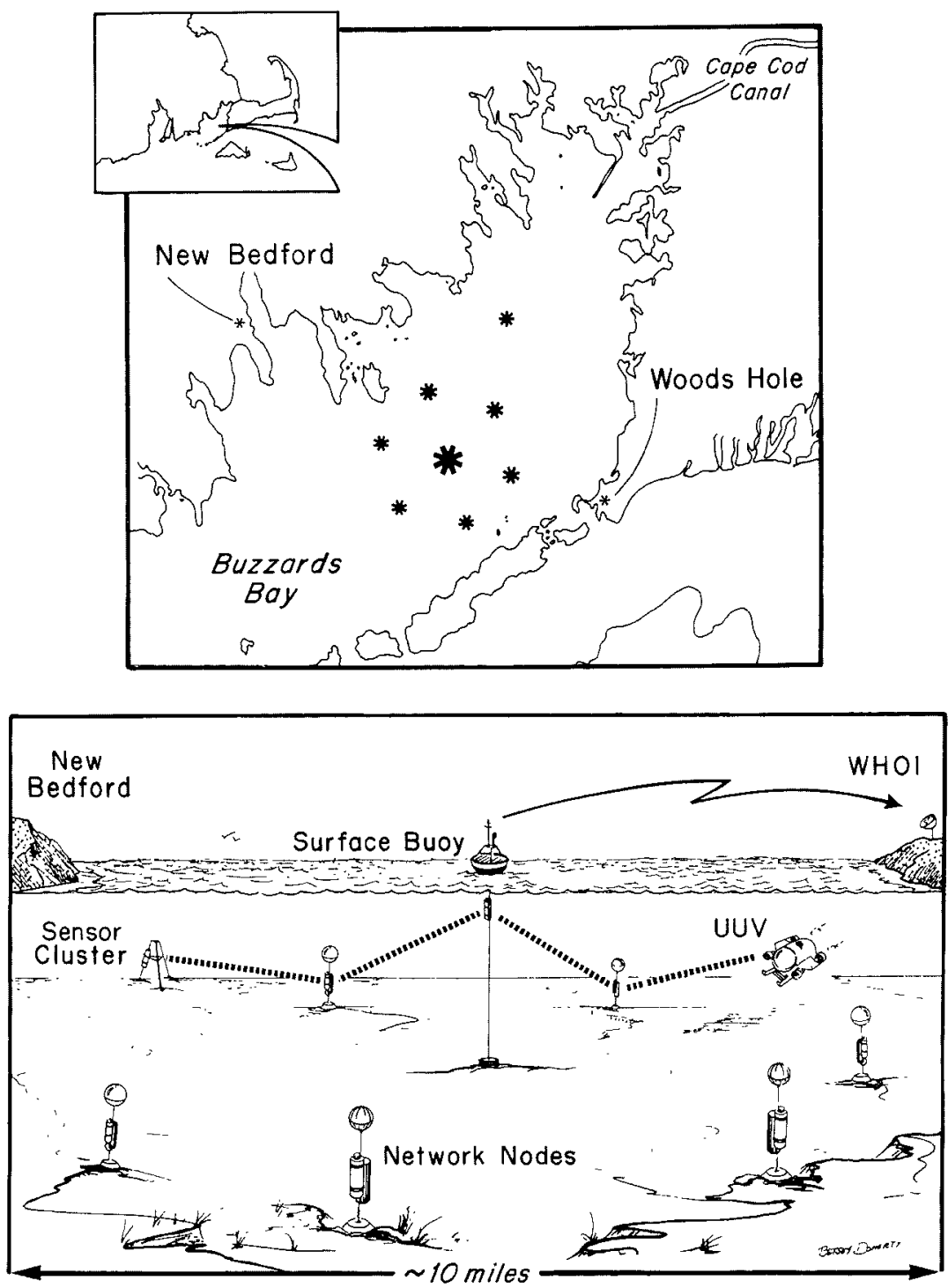

Fig. 8: Schematic of an Acoustic Local Area Network (ALAN) to be deployed on the continental shelf. A variety of sensors are connected to Internet enabling real time access.

vances in battery technology required by the auto industry are expected.

Because propulsion typically consumes a large fraction of the power required by an AUV, improvements in propulsion efficiency pay dividends. Both propeller- and buoyancy-driven vehicles will be used in future AOSNs. Propeller propulsion systems are relatively unreliable. Propeller-driven vehicles lose efficiency to electric motor losses, gearbox friction, shaft seal friction. viscous losses, and the hydrodynamic inefficiency of the propeller. Advanced propulsion techniques with flapping foils in which the vehicle body dynamically interacts with the fin are the subjects of research (Triantafyllou et al., 1993).

\section{Materials and Fabrication}

AUVs should be close to neutral buoyancy to minimize energy expended in maintaining or changing depth. Because the objective is to build small, easy to handle vehicles, methods of obtaining buoyancy without incurring a weight penalty are attractive. An alternative to syntactic foam (glass microspheres imbedded in epoxy) is a pressure housing with net buoyancy greater than that needed for the electronic subsystems. Composite materials (e.g., carbon fiber epoxy) have been used for pressure housings for deep ocean vehicles (Walton, 1991). Fabrication and repair remain areas of research. Advanced materials and computer aided manufacturing will drive down AUV unit cost.

\section{Implementation}

The implementation strategy is to capitalize on state-of-the-art technology and proceed systematically in a series of well-defined increasingly complex basic and applied missions. Critical in this
Both propeller- and bouyancy-driven vehicles will be used in future AOSNs. 
process are the experience and feedback gained from specific field experiments at each step. An experiment is currently scheduled in the Arctic for spring 1994. Objectives include high-resolution underice morphology and thickness mapping for input into sea ice constitutive laws and fracture mechanics models. This initial deployment will consist of a single-node vehicle/multinode telemetry system. After the Arctic effort, an experiment is planned on the continental shelf to rapidly characterize the coastal regime for input into short-term ocean forecasting models and environmental quality assessment. Potential future experiments include coastal mixing and deep ocean convection. Community involvement is essential, and investigators who perceive the potential of these new tools to address problems of interest are encouraged to collaborate in defining future directions of development.

\section{Summary}

A principal motivation for the AOSN is economically feasible ocean sampling adequate for rigorous hypothesis testing and long-term monitoring. Recent advances in technology make this possible. An incremental development plan is being pursued that coordinates government, industry, and academic efforts. Success depends on sustaining three processes in parallel:

1. Addressing specific science questions through a series of progressively more complex experiments. In situ experience, persistence, and engineering feedback are critical. Results of these experiments must be published and have an impact on both the oceanographic and nonoceanographic communities.

2. Integrating engineering research with basic and applied science missions. It is important to focus talented design engineers and students on ocean sampling problems. Because the network architecture will be open to enable access by sensors and platforms of opportunity, consensus on some standardization will be necessary.

3 . Collaborating with industry to ensure economical production and service of compatible components that can be networked readily. The AOSN approach depends critically on low unit cost.

The AOSN has the potential to revolutionize ocean sampling by providing the individual investigator with a personal platform and by fostering a new way of thinking in the design and execution of in situ "experiments" utilizing the power of spatial-temporal adaptive sampling and the diversity of network coverage. An intangible aspect of these new tools for ocean science is the interest and excitement they generate. Reminiscent of the most recent class of pioneering geophysical observation platforms, the earth orbiting satellite, AUVs seem to engage a wide range of nonspecialists of all ages. In addition to revolutionizing sampling, their use in stimulating education and rais- ing the popular level of consciousness can also contribute to the future of ocean science.

\section{Acknowledgments}

Funding for various components of this work has been provided by ONR, ARPA, NOAA, MIT-Sea Grant, and NSF.

\section{References}

Abbott, R.C.. 1978: Submersible acoustic navigation for precision underwater surveys, I.E.E.E.M.T.S., Proceedings Oceans $78,462-465$.

Albus, J.S., 1988: System Description and Design Architecture for Multiple Autonomous Undersea Vehicles, NIST Tech. Note 1251.

Bellingham, J.G., C. Goudey, T.R. Consi, and C. Chryssostomidis, 1992: A small long range vehicle for deep ocean exploration, Proceedings, Intern. Offshore \& Polar Engineering Conf., San Francisco, 151-159.

Catipovic, J., D. Brady, and S. Etchemendy, 1994: Development of underwater acoustic modems and networks. Oceanography, 6, 112-119.

Deffenbaugh, M., H. Schmidt, and J.G. Bellingham, 1993: Acoustic navigation for Arctic underice AUV missions, Proceedings, Oceans 93, Victoria, British Columbia.

Dickey, F.R. and J.A. Edward, 1978: Velocity measurement using correlation sonar. In: Position, Location and Navigation Symposium, I.E.E.E. Aerospace and Electronics Systems Society, 255-264.

Elliot, S., and R. Olson, 1984: Vehicle tracking using advanced acoustic technology in an ultrashort baseline system, Proceedings, ROV 84, Mar. Tech. Soc., 42-44.

Hutchison, B.L., 1991: Workshop for Sensor and Navigation Issues for Unmanned Underwater Vehicles, Mass. Inst. Tech. Sea Grant and C.S. Draper Laboratory, Cambridge, MA.

Jacobsen, H.P., R.A. Klepaker, F.T. Knudsen, and K. Vestgard, 1985: A combined underwater acoustic navigation and control system, Proceedings. ROV 85. Mar. Tech. Soc., $52-56$.

Johnstone, R.S., and D.W. Fries, 1988: Simulation of a submerged autonomous vehicle with inertial navigation, DARPA/CSDL Symposium on Modeling and Simulation, $15-17$.

Patrikalakis, N.M., and L. Bardis, 1991: Localization of rational B-spline surfaces, Engineering with Computers, 7. 237-252.

Stasior, W., 1991: Autonomous localization for underwater vehicles. M.S. thesis, Department of Electrical Engineering and Computer Science, Mass. Inst. Tech.

Sternick, L., 1978: Velocity determination by doppler sonar in deep water. Proceedings, I.E.E.E. PLANS 1978 , 265-271.

Triangafyllou, M.S. and K. Streitlien, 1991: Distributed control of multiple AUV's forming effective chains. Proceedings. Seventh Int. Symp. on Unmanned Untethered Submersibles Technology, 499-518.

Triantafyllou, G.S., M.S. Triantafyllou, and M.A. Grosenbaugh, 1993: Optimal thrust development in oscillating foils with application to fish propulsion. J. Fluids Structurcs. 7, 205-224.

Tuohy, S.T., N.M. Patrikalakis, J.J. Leonard, J.G. Bellingham and C. Chryssostomidis, 1993: AUV navigation using geophysical maps with uncertainty, 8th Int. Symp. on Unmanned, Untethered Submersible Technology, 265-276.

Turner, R.M., J.S. Fox, E.H. Turner, and D.H. Blidberg, 1988: Multiple autonomous vehicle imaging system (MAVIS), Proceedings, Sixth Int. Symp. on Unmanned Untethered Submersibles Technology, 526-536.

Walton. J.M.. 1991: Advanced unmanned search system, Proceedings, Oceans 91, I392-1399. $\square$ 